\title{
Extraction and Analysis of the P Peak Morphology
}

\author{
Priyanka Mayapur ${ }^{1}$ \\ B.E Student, Dept. Of Electronics and Communications Engineering, \\ Agnel Institute of Technology and Design, Assagao, Goa, India ${ }^{1}$
}

\begin{abstract}
An Electrocardiogram (ECG) could be defined as a continuous recording of electrical signals of the heart against time. Analysis of ECG by identifying the various features and traits could help us detect the various cardiac peculiarities. Automatic classification of ECG has evolved as an emerging tool in medical diagnosis for effective treatments. The work proposed in this paper has been implemented using MATLAB that presents an algorithm to detect the various features and the possible abnormalities it could represent. ECG signals in this work are collected across various databases. The processing of the data was done on the Lead-II ECG signals. In addition to that, this paper also provides a comparative study of various methods proposed by researchers used to detect and evaluate $\mathrm{P}$ peaks thus helping us obtain the results accurately, thus enabling precise calculations of the waveforms.
\end{abstract}

Keywords: Electrocardiogram (ECG), Lead-II Configuration, P waves, Heart Defects, Matlab.

\section{INTRODUCTION}

ECG signals are highly non-stationary signals whose statistical properties change over time. ECG is an advanced recording method of bioelectric signal originated in the heart which records the electrical impulses of the heart for diagnostic purposes $[2,3]$. It provides valuable information about the activity of the human heart and is used to measure the rate, rhythm, regularity of the heartbeats, analyze the position, thickness and the size of the chambers of the heart, any heart defect or inflammation and its respective symptoms, effects of drugs or mechanical devices to regulate the heart $[2,4]$.

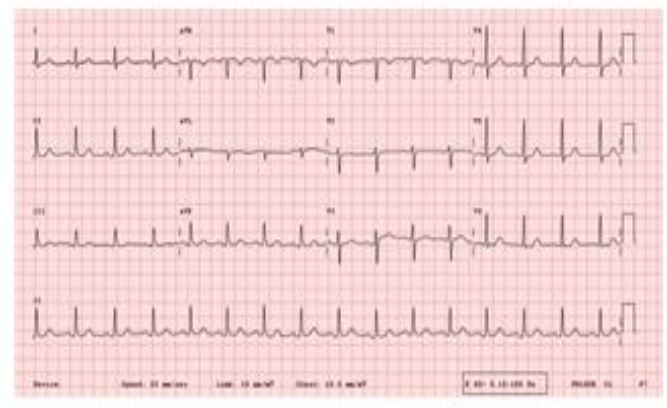

(a)

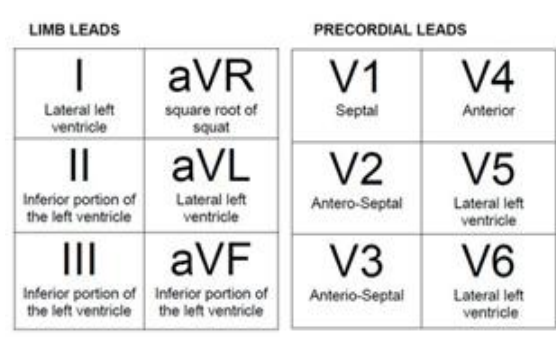

(b)

Fig. 1 A 12 channel recording ECG (a) and ECG Lead Interpretations

It was invented by Willem Einthoven in 1901 while he was working in Leiden, Netherlands. Usually the ECG is recorded in an image consisting of all 12 channels or lead recordings interlaced 3 second intervals from combinations of leads per row. They often occur in the same order (First row: I, AVR, V1, V4; Second row: II, AVL, V2, V5; Third row: III, AVF, V3, V6) all occurring aligned in columns. Since distinct diseases manifest differently in each of the leads, it is important to isolate the different leads involved. Accurate measurements of ECG parameters are an important requirement for ECG analysis and this could be done using signal processing. It's basically a technique used to extract the morphological and dynamic features in order to classify and evaluate an ECG.

\section{THE HEART AND THE CARDIAC CYCLE}

Basically the heart is composed of four chambers, the upper two chambers being the atria are responsible to receive blood from the body and the lower two chambers being the ventricles are responsible for contracting and pumping the blood through the entire body [2]. The whole ECG signal recording is a combination of several consecutive cardiac 
Vol. 7, Issue 11, November 2018

cycles that results due to the depolarization and repolarization of the ions in the blood which include a fairy period of waves and peaks corresponding to the consecutive heart action phases [5].

The sinus (Sino-atrial node) node located near the entrance of the superior vena cava vein, acts as a generator of the sinus rhythm that produces the heart frequency at about 60-100 cycles per minute. This activation is then propagated to the right and left atria muscle tissues. There is a delay at the atrioventricular node, to allow the ventricles to fill with blood from atrial contraction. This is then followed by the depolarization propagating to the ventricles through the Bundle of His which spreads along the Purkinje fibers. This in turn activates the ventricles that contract and pump blood to the aorta and to the rest of the body. Finally, depolarization occurs followed by repolarization and this cycle is repeated [6]. The track of each heartbeat would consist of several waves/ peaks, segments, intervals and joints as a recurrent wave sequence as shown in the figure below in Fig $2(\mathrm{~d})$.

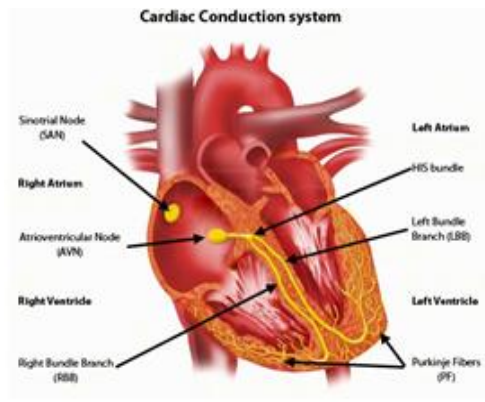

(c)

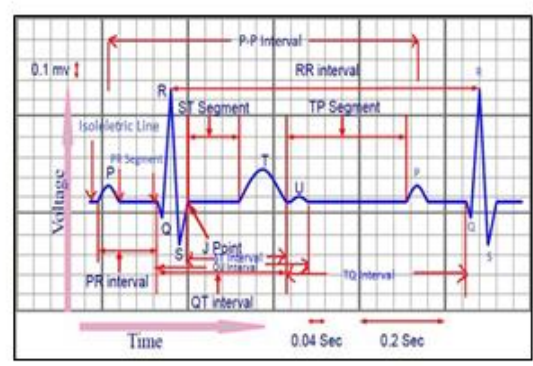

(d)

Fig. 2 The Human Heart and A General ECG Waveform

The table 1 shows the ECG features and descriptions.

Table 1: ECG Features and their Description

\begin{tabular}{|c|c|}
\hline FEATURE & DESCRIPTION \\
\hline P WAVE & P-waves represent atrial depolarization. \\
\hline Q WAVE & $\begin{array}{l}\text { The normal Q wave represents septal depolarization and is any initial downward } \\
\text { deflection after the } \mathrm{P} \text { wave. }\end{array}$ \\
\hline R WAVE & $\begin{array}{l}\text { The } \mathrm{R} \text { wave represents early ventricular depolarisation and is normally the easiest } \\
\text { waveform to identify on the ECG. }\end{array}$ \\
\hline S WAVE & $\begin{array}{l}\text { The first negative deflection after the } \mathrm{R} \text { wave represents the } \mathrm{S} \text { wave indicating the late } \\
\text { ventricular depolarization. }\end{array}$ \\
\hline T WAVE & The T-wave represents ventricular repolarization. \\
\hline U WAVE & $\begin{array}{l}\text { U waves represent re-polarization of the Purkinje fibers that indicates the last remnants } \\
\text { of the ventricular repolarization. Generally it is } 0.05 \mathrm{mV} \text { and has duration of } 0.1 \mathrm{~s} \text {. }\end{array}$ \\
\hline $\begin{array}{l}\text { P-R SEGMENT OR } \\
\text { PQ SEGMENT }\end{array}$ & $\begin{array}{l}\text { The PR or PQ segment is the flat, usually isoelectric segment between the end of the P } \\
\text { wave and the start of the QRS complex. This segment represents the time the impulse } \\
\text { takes to reach the ventricles from the sinus node. }\end{array}$ \\
\hline $\begin{array}{l}\text { P-R INTERVAL } \\
\text { OR PQ INTERVAL }\end{array}$ & $\begin{array}{l}\text { The time taken for electrical activity to move between the atria and ventricles is } \\
\text { represented by this interval. }\end{array}$ \\
\hline R-R INTERVAL & $\begin{array}{l}\text { The RR-interval begins at the peak of one } \mathrm{R} \text { wave and ends at the peak of the next } \mathrm{R} \\
\text { wave and represents the time between two QRS complexes. }\end{array}$ \\
\hline P-P INTERVAL & It indicates the duration of atrial cycle (atrial rate). \\
\hline QRS COMPLEX & The depolarization of the ventricles is represented by the QRS Complex. \\
\hline QT INTERVAL & It represents the time taken for the ventricles to depolarize and then repolarize. \\
\hline ST SEGMENT & $\begin{array}{l}\text { The isoelectric line that represents the time between depolarization and repolarization } \\
\text { of the ventricles (i.e. contraction) represents the ST segment. }\end{array}$ \\
\hline J-POINT & $\begin{array}{l}\text { The J point is the junction between the termination of the QRS complex and the } \\
\text { beginning of the ST segment. }\end{array}$ \\
\hline T-P INTERVAL & $\begin{array}{l}\text { The isoelectric interval on the electrocardiogram (ECG) is TP segment that represents } \\
\text { the time when the heart muscle cells are electrically silent. }\end{array}$ \\
\hline T-Q INTERVAL & Termed as the diastolic interval through the ECG. \\
\hline Q-U INTERVAL & $\begin{array}{l}\text { The QU interval is a measure of the time between the start of the Q wave and the end } \\
\text { of the U wave in the heart's electrical cycle. }\end{array}$ \\
\hline
\end{tabular}




\section{THE CARDIAC ABNORMALITIES}

We all know that heart diseases are recognized as one of the major causes of death in the world and the best diagnostic tool to determine any abnormality in the cardiac function or tissue damage would be through ECG [7]. In the morphology of ECG signal where the normal rhythm of the heart represents no disease or disorder is called Normal sinus rhythm (NSR). Cardiac Arrhythmia could be defined as a disorder or disturbance or any abnormality resulting in the normal activation sequence of the myocardium giving rise to irregular heartbeat or abnormal rhythm of the heart that may cause permanent injury to the heart. Although cardiac arrhythmia is one of the leading causes of death, it can be treated if detected on time [8, 9 and 10]. Under the expert guidance of the doctors and after lots of literature review, it was seen that Lead II is the most preferred monitoring lead of choice for continuous ECG monitoring. Nowadays, ECG has become a golden medium for detecting Arrhythmia and Cardiovascular diseases and also could detect bifid P wave in lead II (P Mitrale).

\section{THE P WAVE MORPHOLOGY IMPORTANCE}

The ECG deflection is said to be in rhythm if the tracing follows the following sequence usually composed of these components; P wave followed by QRS Complex followed by T wave and then $\mathrm{U}$ wave. $\mathrm{U}$ wave is normally invisible in 50 to $75 \%$ of ECGs because it is generally hidden by the $\mathrm{T}$ wave and the upcoming new $\mathrm{P}$ wave but in our processing procedure, it is skipped for further analysis [2].

The $\mathrm{P}$ wave represents atrial activation (contractions), generally termed as a summation wave, generated during the depolarization front while transiting towards the left and the right atrium. P waves are very clearly visible in Lead-II and V1 (biphasic) during sinus rhythm. On this count, it is always positive and upright in Lead-II and AVF configuration and negative in AVR configuration during sinus rhythm. P waves could be considered as the key factor in determining whether a patient is in sinus rhythm or not. A normal sinus $\mathrm{P}$ wave present immediately before every regular QRS complex indicates that the electrical impulse responsible in generating the $\mathrm{P}$ wave originated in the SA node itself and that normal de-polarization of the atrium has occurred. If there are no $\mathrm{P}$ waves, then the exact rhythm and rate needs to be found out, this could be an indication of cardiac abnormality. Also the P waves observed in a normal sinus rhythm are round and smooth, any other sign found to be hampering this shape like saw-tooth, peaked, prolonged, bifid, notched or inverted in Lead-II, could again indicate a cardiac abnormality. It would be termed as ectopic if the $\mathrm{P}$ wave has morphology different from the typical sinus morphology which would mean coming from somewhere other than the sinus node. In addition to this, it could also shed some light on enlargements of the atria. Also processing the segments and intervals related to $\mathrm{P}$ Wave are done. The $\mathrm{P}$ wave itself in a whole provides a lot of information about the health of the heart and it is very necessary to determine and analyze it in order to be aware of diseases if any and also the functioning of the heart along with its rhythm. Processing this peak could really provide us some significant information and treat the defects.

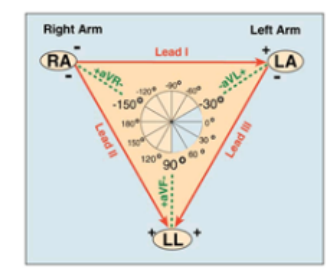

(e)

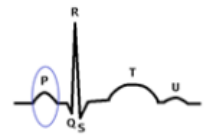

(f)

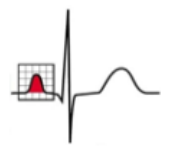

(g)

Fig. 3 Lead-II ECG Configuration (e) and the Normal P Wave (f and g)

\section{THE LITERATURE SURVEY}

In a paper proposed by Namitha Thomas et al, the detection and delineation of $\mathrm{P}$ and $\mathrm{T}$ wave using an ECG signal has been done using the proposed methodology where they have first detected the QRS Complex using the Pan Tompkins algorithm and eliminated the frequencies using the Linear filters. The pre-processed ECG signal and the extracted wave search region are then applied to the partially collapsed Gibbs sampler. To determine P and T-waves from collapsing samples they used a Partially Collapsed Gibbs Sampler (PCGS). The output of PCGS is then applied to Wave Indicator Estimation, amplitude estimation and amplitude estimation block. The wave indicator was done by using Local Map A Posteriori (MAP) method. The amplitude estimation is done by using fuzzy theory. The waveform estimation is done by using neural network. The estimation of noise variance of the wave is done by using MMSE method [1].

In a paper proposed by Chao Lin et al, The delineation of $\mathrm{P}$ and $\mathrm{T}$ waves were done using a sequential Bayesian detection-estimation algorithm for simultaneous $\mathrm{P}$ and $\mathrm{T}$ wave detection, delineation, and waveform estimation on a beat-to-beat basis. Their method was based on a dynamic model which exploits the sequential nature of the ECG by 
introducing a random walk model to the waveforms. The core of the method is a marginalized particle filter that efficiently resolves the unknown parameters of the dynamic model. The proposed algorithm was evaluated on the annotated QT database and compared with state-of-the-art methods. Its on-line characteristic is ideally suited for realtime ECG monitoring and arrhythmia analysis [13].

V.S. Chouhan et al proposed a method of detecting $\mathrm{P}$ and $\mathrm{T}$ waves by first filtering the signal followed by detecting QRS Complex by using the QRS detection algorithm. In the proposed algorithm, the feature extraction for detection of $\mathrm{P}$ - and T-waves uses computation of gradient, in a sliding window; to ensure sufficient magnitude of the extracted feature, so as to meet the thresholding needs, the proposed algorithm extracts multiple feature components and combines them to attain the final feature signal and also using the sampling instants [15].

Zeeshan Ahmad et al proposed a paper where in $\mathrm{P}$ and $\mathrm{T}$ Peak parameters were detected using an algorithm. In the first stage, they processed the QRS complex using dynamic thresholding technique. The second stage processed $\mathrm{P}$ and $\mathrm{T}$ waves using Elgendi's algorithm depending upon the information extracted from first stage [16].

In a paper proposed by Chao Lin et al, a Bayesian detection-estimation algorithm was used for simultaneous detection, delineation, and estimation of $\mathrm{P}$ and $\mathrm{T}$ waves. A block Gibbs sampler exploited the strong local dependencies in ECG signals by imposing block constraints on the $\mathrm{P}$ and $\mathrm{T}$ wave locations [17].

In a paper proposed by $\mathrm{H}$. K. Chatterjee et al, illustrated a technique for real time detection of $\mathrm{P}$ and $\mathrm{T}$ wave peaks from ECG signal. The technique was implemented on Xilinx field programmable gate array. The characterization of $\mathrm{P}$ and $\mathrm{T}$ wave was also done during the training period [18].

\section{STEPWISE METHODOLOGY}

\section{Step 1: Collection of the ECG Database}

The ECG signals were collected from variety of databases like the MIT-BIH (The Massachusetts Institute of Technology- Beth Israel Hospital Arrhythmia Database), AHA (The American Heart Association ECG Database), ESC (The European Society of Cardiology ST-T Database) and UCI (Machine Learning Repository). The database consisted of several different ECG format waveforms like .mat, .csv, .xml, .dat or .txt. The collections of databases were done from the database banks, ECG Simulators, ECG Machines along with an ECG Amplifier in practical laboratories and Electrocardiographs from the hospitals.

\section{Step 2: Initialization of the ECG Signal}

In order to process an ECG signal, we first need to read and plot the signal. Our Project has been implemented using the multipurpose tool i.e. the MATLAB Environment. If the signal is raw, which usually is unless it's taken from a filtered database, we need to perform initialization and remove the base and gain by using the following formula:

Where $\mathrm{Xi}=$ ECG Sample

$$
\mathbf{X i}=\frac{\mathbf{X i}-\text { Base }}{\text { Gain }}
$$

Base $=$ Baseline Value

Gain= Gain Factor

Once done, we can proceed to reading and plotting of the signal on Matlab. Depending upon the various formats, some signals could be plotted directly (.mat) and some required conversion from one format to the required format ((.csv, .$x \mathrm{ml}$, dat or .txt) to .mat) by choosing the appropriate frequency and threshold along with re-dimensioning of the variable matrix.

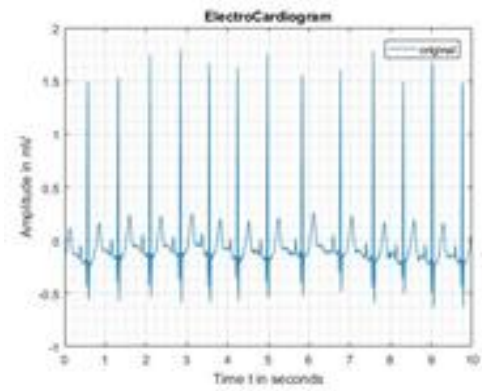

(h)

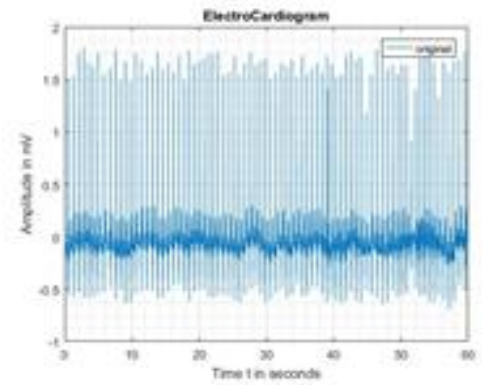

(i)

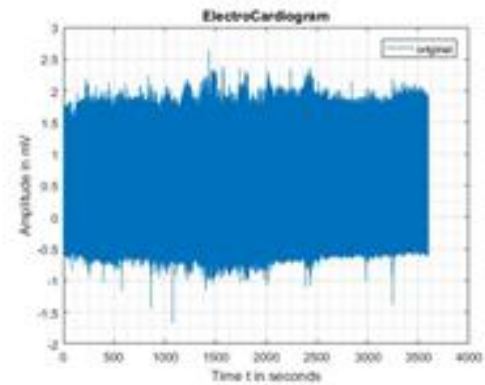

(j)

Fig 4: Original ECG Signals (10, 60 and 3600 seconds) (Normal) (h,I,j resp) 

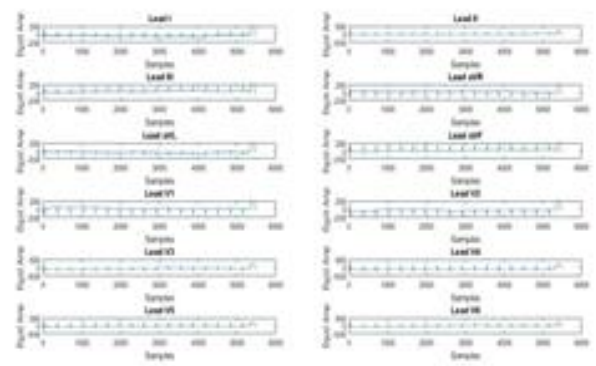

(k)

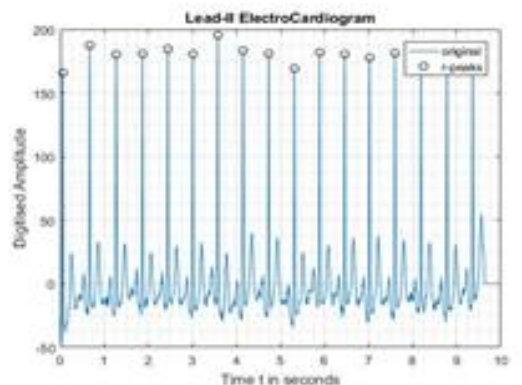

(1)

Fig. 5 Plotting of 12 Lead Configuration ECG Signal from the .xml format to .mat signal (k) and A Lead-II ECG Configuration extracted from 12 Lead Configuration ECG signal (l)

\section{Step 3: Preprocessing Phase}

In the preprocessing stage, the noise is removed or suppressed using specific filters in order to extract the required information from the signal and for noise reduction. This could be done either by performing Amplitude Normalization where in each sample of signal is divided from max of absolute value of signal in order to limit signal dynamic range from -1 to 1 , i.e.

Where $\mathrm{xi}=\mathrm{ECG}$ Sample at a point

$$
\text { Variable }=\frac{x i}{\max (|x|)}
$$

\section{$\mathrm{x}=\mathrm{ECG}$ Sample}

The .mat format signal could be directly plotted in Matlab using a specific command. Considering the .csv and .dat format signals, Conversion and Zero Phase Filtering were done in order to plot it. In case of the .xml format signal, the same procedure was carried out in order to plot the signal which represented all the 12 Lead Configurations followed by extracting the required signal configuration needed to work on (Lead-II).



(m)

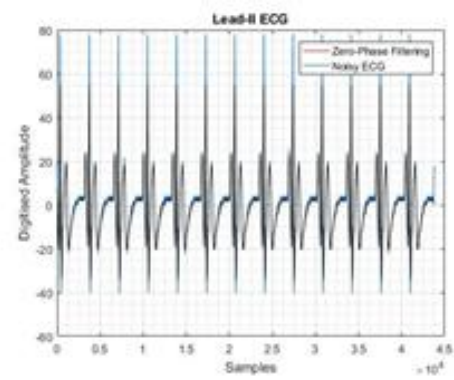

(n)

Fig. 6 Zero Phase Filtering of Extracted Lead-II ECG Signal to (.mat) from (.xml) (m) and (.mat) from (.csv) (n)

\section{Step 4: Feature Extraction and Evaluation}

The feature extraction stage is used to extract diagnostic information from the ECG signal.

Feature extraction and evaluation can be either done to find out:

- $\quad$ Morphological Features

- Dynamic Features

Morphological Features would mean determining the size, shape and structure of the ECG signal including the fiducial points like the peak points, onset and offset (wave boundaries), segments and interval durations.

Dynamic features would mean extracting RR interval, PP interval features, Heart rate, HRV and the R/P ratio.

In this paper, what we present to propose is to extract and analyze the $\mathrm{P}$ peaks along with some of the dynamic features in order to evaluate an ECG attributes properly.

i. So in order to begin with this we first identified the QRS Complex which would help us identify the R peak using the Pan Tompkins Algorithm. R peak detection is a good start for the identification procedure as it is the sharpest component with respect to all the other peaks in a Normal Lead-II ECG Signal and is easier to detect.

ii. In the Pan Tompkins Algorithm, ECG was first filtered using a band pass filter followed by differentiating the signal in order to get the slope information. This was then followed by squaring the signal which made the entire signal values positive concluding it with moving window integration which was done to obtain the waveform feature information. After moving window integration, thresholding of the obtained signal was done. If a peak exceeded the threshold during the first step of analysis, it was classified as a QRS peak (Complex). 
iii. This was then followed by calculating the R-R Interval using the R-Spike Detection Method which is basically calculating the interval between one R-Spike and the next R-Spike (successive R's). This was then used to calculate the heart rate which could be defined as how fast the person's heart could beat in a minute. Initially the mean value of the R-R Interval is calculated and then this duration is then divided into 60. The resulting equation would be:

$$
\text { Rate }=\frac{60}{R-R \text { Interval (Avg) }}
$$

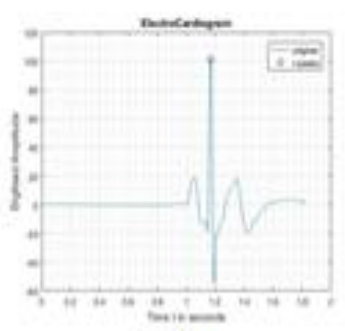

(0)

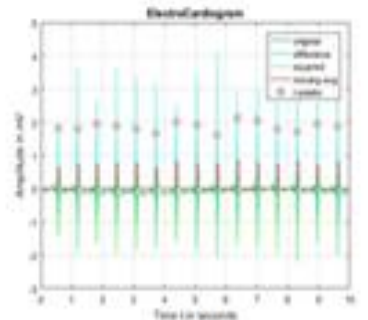

(p)

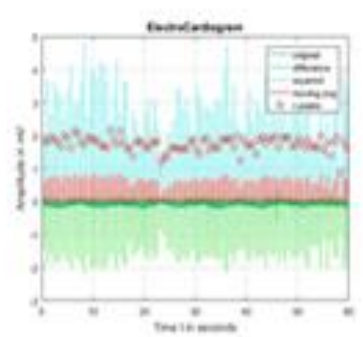

(q)

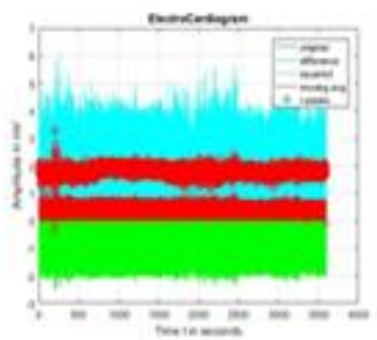

(r)

Fig. 7 Detecting R Peak (o) and QRS Complex using Pan Tompkins Algorithm (10s, 60s, 3600s) (p, q, r resp.)

iv. Using the Moving Window Integration technique along with the Threshold Detection method, we could detect the $P$ peaks in the ECG signal along with their amplitude and locations. This was followed by then determining the wave boundaries of the $\mathrm{P}$ wave, i.e. the onset and the offset named as Pon and Poff resp. So the $\mathrm{P}$ wave duration could be found using the following equation;

$$
\text { P Wave Duration }=\text { Poff }- \text { Pon }
$$

$\mathrm{v}$. The next step was to detect the $\mathrm{Q}$ peak by finding out the first local minimum from the left of the positive $\mathrm{R}$ wave and the onset of the Q peak; Qon, using the same thresholding technique.

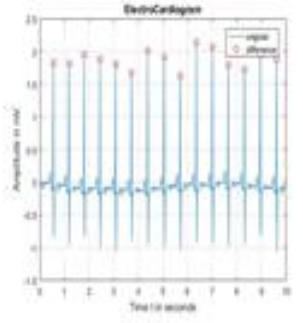

(s)



(t)

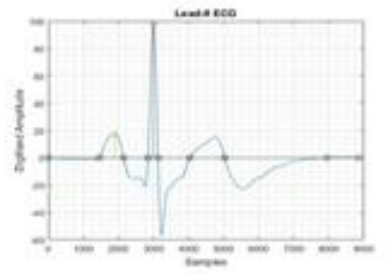

(u)

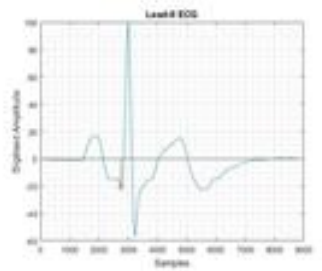

(v)

Fig. 8 Evaluating the R-R interval and the fiducial features (s), Detection of P Peak (t), Wave Boundaries $(\mathrm{u})$ and Q Peak (t)

vi. Once this was done, the PR or PQ Segment was calculated using the equation;

$$
\text { PR Segment }=\text { Qon }- \text { Poff }
$$

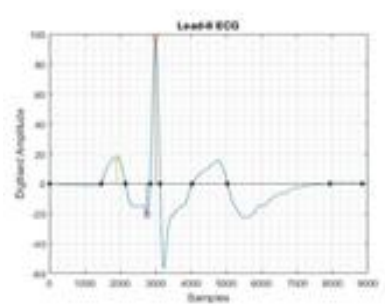

(w)

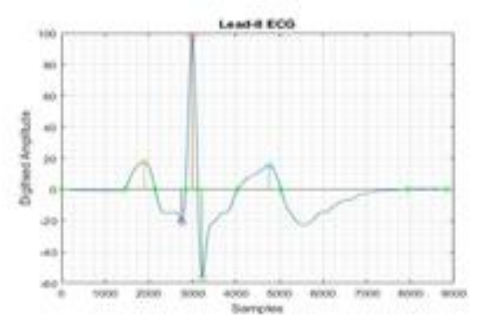

(x)

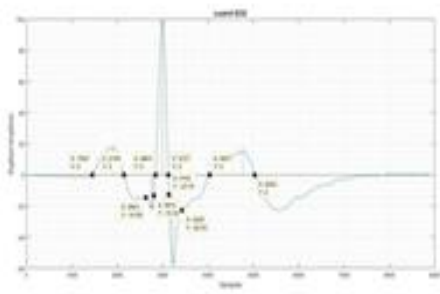

(y)

Fig. 9 Detection of the Fiducial Features (w), Peak Points and Locations;

P, Q, R, S, T Waves (x) and evaluating the Dynamic Features like the PR Segment and Interval (y) 
vii. This was followed by calculating the PR or PQ Interval using the equation;

viii. The $\mathrm{R}$ to $\mathrm{P}$ ratio was computed by using

$$
\text { PR Interval = Qon }- \text { Pon }
$$

$$
\frac{\mathbf{R}}{\mathbf{P}}=\frac{\mathbf{R} \text { Peak (Amplitude) }}{\text { P Peak (Amplitude) }}
$$

ix. Also additionally $\mathrm{S}$ and $\mathrm{T}$ peaks were detected in order to help identify the rhythm using the thresholding technique.

\section{RESULTS}

The table displayed below gives the value of the average of more than 80 samples taken and analyzed in Matlab and that could be considered as Normal ECG based on the characteristics observed.

Table 2: ECG Signal Features and their Respective Values (Normal)
\begin{tabular}{|c|c|c|}
\hline FEATURES & \multicolumn{2}{|c|}{ VALUES } \\
\hline General Factors & \multicolumn{2}{|c|}{ Values } \\
\hline Heart Rate & \multicolumn{2}{|c|}{$60-100$ bpm* } \\
\hline R-R Interval & \multicolumn{2}{|c|}{$0.6^{*}$ s to $1.2^{*}$ s } \\
\hline R to P Ratio & \multicolumn{2}{|c|}{$3^{*}$ to $12^{*}$} \\
\hline Waves & Amplitude(mV) & Duration(s) \\
\hline P Wave & $0.1^{*}-0.35^{*}$ & $0.07^{*}-0.12^{*}$ \\
\hline Q Wave & $0 . *_{-}^{*}-0.3^{*}$ & $<0.04^{*}$ \\
\hline R Wave & $0.8^{*}-1.5^{*}$ & $0.035^{*}-0.09^{*}$ \\
\hline Segments/Intervals & \multicolumn{3}{|c|}{ Duration(s) } \\
\hline PQ or PR Segment & \multicolumn{3}{|c|}{$0.04^{*}-0.12^{*}$} \\
\hline PQ or PR Interval & \multicolumn{2}{|c|}{$0.1^{*}-0.2^{*}$} \\
\hline QRS Complex & \multicolumn{2}{|c|}{$0.06^{*}-0.12^{*}$} \\
\hline
\end{tabular}

- $\quad$ *These obtained values in the table are calculated manually as well as using specific algorithms through computer processing in Matlab by analyzing more than 80 samples and is verified by doing a lot of literature review and is approved by the doctors.

- $\quad$ The entered values in the table above are the average values of more than 80 samples after processing.

Any value or feature that does not fall into the criteria and has a haphazard shape that does not have regularity and rhythm as defined in table 2 would be considered as an abnormal ECG.

After processing 10 such similar signals, it was seen that these signal tracings followed the particular sequence, a $\mathrm{P}$ wave with a round shape followed by a regular QRS Complex followed by $\mathrm{T}$ wave. Also signal in Fig. 10a maintains a heart rate within 60 to $100 \mathrm{BPM}$ at rest along with the specific values of the features as obtained in the table. After a lot of literature review and processing, it could be learnt that the following traits correlate to a normal sinus rhythm criteria and therefore, it could be said that the subject could be in Normal Sinus Rhythm. After processing 10 such similar signals, it could be seen that this signal lacks the necessary sequence and therefore, could be stated that this subject does not have a Regular Rhythm and few of the Waves, Segments and Intervals are either absent or immeasurable, P wave being one of them. The Rate observed in Fig. 10b is 67 BPM at rest. This represents an ABNORMAL ECG Signal. Since few features are indiscernible along with a Chaotic Rhythm and the QRS Complex duration is $0.0842 \mathrm{~s}$, from the literature review, it could be concluded that this could represent ATRIAL FIBRILLATION.

After processing 10 such similar signals, it could be seen that this signal lacks the necessary sequence and therefore, could be stated that this subject does not have a Regular Rhythm and the Waves, Segments and Intervals are either absent or immeasurable, $\mathrm{P}$ wave being difficult to locate. The Rate observed in Fig. 10c is above 100 BPM. This represents an ABNORMAL ECG Signal. Since few features are indiscernible along with a Chaotic Rhythm, from the literature review, it could be concluded that this could represent ATRIAL FLUTTER.

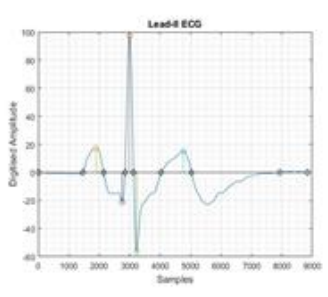

(a)

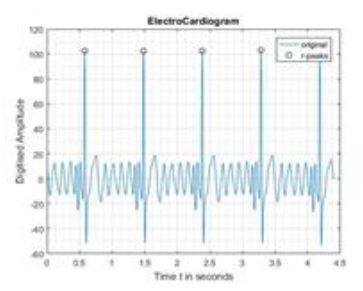

(b)

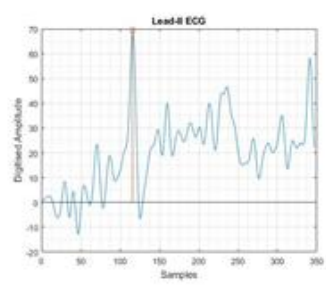

(c)

Fig. 10 ECG in Normal Sinus Rhythm (a), Missing P Peak Plotted Waveform (b), Chaotic Plotted Waveform (c) 
The ECG Analysis after processing 10 signals of similar kind shows that it does not have a Regular Rhythm and few of the Waves, Segments and Intervals are either absent or immeasurable. The Rate observed in Fig. 11d is 118 BPM at rest. This represents an ABNORMAL ECG Signal. Since few features are indiscernible with the absence of $\mathrm{P}$ waves and the QRS Complex duration is $0.5296 \mathrm{~s}$, which is wide and bizarre and from the literature review, it can be concluded that this could represent VENTRICULAR TACHYCARDIA.

The ECG Analysis after processing 10 signals of similar kind states that it does have a Regular Rhythm and the Waves, Segments and Intervals are measurable. The Rate observed in Fig. 11e is 84 BPM at rest. It has a bifurcated P Wave, it can be concluded that this could represent P MITRALE. This represents an ABNORMAL ECG Signal.

Following figures represent several other signals with a chaotic rhythm and immeasurable segment and interval and with a missing $\mathrm{P}$ wave in few, one of which is represented in Fig. 11f.

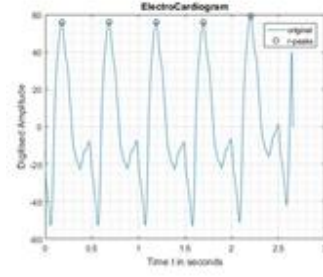

(d)

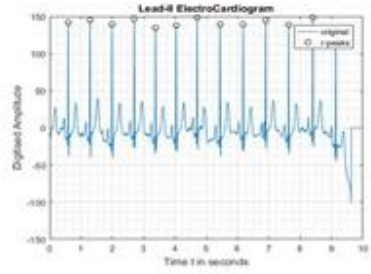

(e)

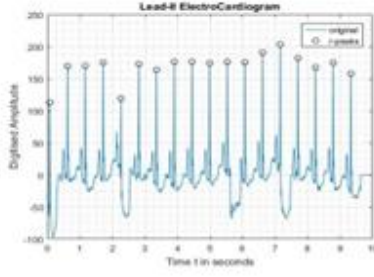

(f)

Fig. 11 Chaotic Plotted Waveforms (d, e, f resp)

\section{CONCLUSION}

Biomedical signals are non-stationary signals whose analyses require better time and frequency resolution. Such analysis include de-noising, filtering, normalizing, squaring, averaging, encoding, decoding, compressing, decompressing, deinterleaving, constructing, reconstructing and comparing of the data. In consultation with the cardiologists and after a lot of processing and analyzing, it was seen that Lead-II ECG Configuration can detect Arrhythmias and P Mitrale. The results obtained from our project cannot be immediately applied to the population. Many of our subjects suffered from a combination of heart defects. Classification and Detection of the heart defects using Lead-II configuration requires many more samples. Future research heading in this direction is necessary with a larger sample size in order to accurately pinpoint the various heart defects individually.

\section{ACKNOWLEDGEMENT}

We were really privileged to be guided by several doctors and technicians along with our college of Agnel Institute of Technology and Design. Thanks are also addressed to the field team and to the anonymous reviewers as well.

\section{REFERENCES}

[1]. Namitha Thomas, D.J.Jagannath, "DETECTION OF PEAK AND BOUNDARIES OF P AND T WAVES IN ECG SIGNALS", International Journal of Electronics Signals and Systems, Vol-3, Iss-1, 2013.

[2]. Anand Kumar Joshi, Arun Tomar, Mangesh Tomar, "A Review Paper on Analysis of Electrocardiograph (ECG) Signal for the Detection of Arrhythmia Abnormalities", International Journal of Advanced Research in Electrical, Electronics and Instrumentation Engineering, Vol. 3, Issue 10, October 2014, ISSN: 22788875.

[3]. Sonal Pokharkar, Amit Kulkarni , "ECG Real Time Feature Extraction Using MATLAB", International Journal of Technology and Science, Volume V, Issue 1, 2015 pp. 1-4.

[4]. Rajan Chaudhary, Anand Prakash, Chandan Gupta, "Comparison of Different ECG Signals on MATLAB", International Journal of Electronics and Computer Science Engineering, Volume2, Number 2.

[5]. A.Peterkova, M. Stremy, "The raw ECG signal processing and the detection of QRS complex", IEEE European Modelling Symposium, 2015 IEEE.

[6]. S. Thulasi Prasad, S. Varadarajan, "Analysis of ST Segment Abnormalities in ECG using Signal Block Averaging Technique”, International Journal of Advanced Research in Computer and Communication Engineering, Vol. 4, Issue 2, February 2015.

[7]. Er. J.S Dhir, Er. N.K. Panag, "ECG Analysis and R Peak Detection Using Filters and Wavelet Transform", International Journal of Innovative Research in Computer and Communication Engineering, Vol. 2, Issue 2, February 2014.

[8]. Mr. Vyankatesh S. Thorat, Dr. Suresh D. Shirbahadurkar, Mrs. Vaishali V. Thorat, "Study and Literature Survey for Classification of Cardiac Arrhythmia With Respect to ECG and HRV Signal", International Journal of Advanced Research in Computer and Communication Engineering, Vol. 5, Issue 4, April 2016.

[9]. Preeti Raman, DR SM Ghosh, "Classification of Heart Diseases based on ECG analysis Using FCM And SVM Methods", International Journal of Engineering Science and Computing, 2016.

[10]. Sandeep Sharma (Scholar), Prof. Vikas Mittal, Prof. Yuvraj Sharma, "Detection of ECG points using Principal component analysis", International Association of Scientific Innovation and Research in Association with International Journal of Emerging Technologies in Computational and Applied Sciences (IJETCAS), 13-190, ISSN:2279-0055. 
[11]. Adam Szczepanski, Khalid Saeed, and Alois Ferscha, “A New Method for ECG Signal Feature Extraction”, L. Bolc et al. (Eds.): pp. 334341, 2010, Springer-Verlag Berlin Heidelberg 2010.

[12]. Afseen Naaz, Mrs Shikha Singh, "Feature Extraction and Analysis of ECG signal for Cardiac Abnormalities- A Review", International Journal of Engineering Research \& Technology, Vol. 3 Issue 11, November-2014.

[13]. Chao Lin, Audrey Giremus, Corinne Mailhes, and Jean-Yves Tourneret, "BEAT-TO-BEAT P AND T WAVE DELINEATION IN ECG SIGNALS USING A MARGINALIZED PARTICLE FILTER".

[14]. Ross MacKenzie, "Short PR Interval, JOURNAL OF INSURANCE MEDICINE”, J I n s u r M e d 2005, pp-37:145-152.

[15]. V.S. Chouhan and S.S. Mehta, "Threshold-based Detection of P and T-wave in ECG using New Feature Signal", International Journal of Computer Science and Network Security, VOL.8 No.2, February 2008.

[16]. Zeeshan Ahmad, Ijlal Haider, Omair Bashir Ahmed, Sufyan Ali Raza, Habib-ur-Rehman Khalid, "P and T Peak Parameter Detection in ECG Signals", The 2015Biomedical Engineering International Conference (BMEiCON-2015).

[17]. ChaoLin,GeorgKail,JeanYvesTourneret1,CorinneMailhes, andFranzHlawatsch, "P AND T WAVED ELINEATION AND WAVEFORM ESTIMATION IN ECG SIGNALS USING A BLOCK GIBBS SAMPLER”, Prague, Czech Republic, May 2011, pp. 537-540.

[18]. H. K. Chatterjee, R. Gupta, M.Mitra, "Real time P and T wave detection from ECG using FPGA", Published by Elsevier Ltd, 2012.

[19]. Chao Lin, "P and T Wave Detection in Electrocardiogram (ECG) Signals".

[20]. Bharat K. Kantharia, "P waves in the electrocardiogram recording of tachycardia: 'You can run, but you cannot hide", Europace 2011.

[21]. Sahil Verma, Ramesh Kumar Sunkaria, Barjinder Singh Saini, "Heart Rate Determination with RR Interval and PP Interval Time Series", International Journal of Advanced Research in Computer Science, Volume 3, No. 3, May-June 2012.

[22]. PR Intervals- Fast Easy ECGs - A Self-Paced Learning Program.

[23]. ElectroCardioGraphy-ECG made extra easy.

[24]. Advanced ECG Interpretation.

[25]. JOHN P. DIMARCO, M.D., PH.D. ,Cardiac Electrophysiology : A Cost-Effective Approach.

[26]. The Normal ECG and its (Normal) Variants.

[27]. ECG Primer.

[28]. ECG Monitor, Project Id H3290.

[29]. USB TAP Probe User Guide.

[30]. Signal Processing Toolbox, For Use with MATLAB, Users Guide Version 5.

[31]. P and T Wave Detection in Electrocardiogram (ECG) Signals, Chao Lin, Seminar SC, June 2011.

[32]. INTRODUCTORY GUIDE TO IDENTIFYING ECG IRREGULARITIES, DailyCare BioMedical Inc.

[33]. www.ecgsim.org

[34]. www.lifeinthefastlane.com

[35]. www.researchgate.net

[36]. www.cardionetics.com

[37]. www.wikilectures.eu

[38]. Anika Niambi Al-Shura, Integrative Anatomy and Pathophysiology in TCM Cardiology.

[39]. Malcolm S. Thaller, M.D, The Only EKG Book Youll Ever Need.

[40]. Altunkeser BB, Ozdemir K, Gök H, Temizhan A, Tokaç M, Karabağ T, "Can P wave parameters obtained from 12-lead surface electrocardiogram be a predictor for atrial fibrillation in patients who have structural heart disease?", Vol 54, Issue 4, 2003.

[41]. S. Sovilj; G. Rajsman; R. Magjarevic,"Continuous multiparameter monitoring of P wave parameters after CABG using wavelet detector, IEEE, 06 February 2006".

[42]. S. S. Mehta, S. C. Saxena, and H. K. Verma, "Recognition of P and T waves in electrocardiograms using fuzzy theory," in Proc. 1st Reg. Conf., IEEE Eng. Med. Bio. Soc., New Delhi, India, Feb. 1995, pp. 2/54-2/55.

\section{BIOGRAPHY}

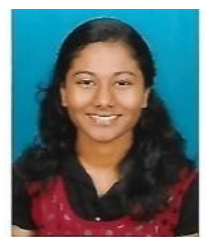

Priyanka Mayapur is a graduate in Electronics and Communications Engineering from AITD, Batch 2017. She has worked as a Project Manager in STEAM Labs and many more places and is an avid enthusiast of Research, Media, Fashion Designing, Astronomy and Teaching and was also a representative of the chapter of AITD for Google Developers Goa and is a Member of the Student Chapter for IETE (The Institution of ETC Engineers). She has also worked on a project named "Analysis of Lead-II ECG for Classification and Detection of Heart Defects." 